\title{
El tiempo de cuidado familiar a mayores dependientes en España: entre la donación y la expropiación del tiempo ${ }^{1}$
}

\author{
Jesús Rogero-García \\ Dpto. de Sociología \\ Universidad Autónoma de Madrid \\ <jesus.rogero@uam.es>
}

\begin{abstract}
Artikulu honetan, aztergai hartu du egileak adindunak etxean zaintzen diren kasuetan lanean ematen den denbora, eta, ebaluatzen saiatu da gaiak eguneroko jardunean zaintzaileenganako izaten dituen eraginak. 2002-2003ko Denboraren Erabileraren Inkesta oinarri harturik, honako galderei erantzuten saiatu da egilea: nola banatzen dute senideek denboraren erabilera menpeko adinduak bizi diren etxeetan? hautatzen al du norberak bere zaintzarako modua? eta zeintzuk eta nolakoak dira zaintzaren eraginak? Azterlan honetan, argiro erakusten da zaintzaren inguruko arduren banaketak desberdintasun-maila jasoa duela. Azterlanaren emaitzen arabera, zaintzaileek etxeko beharrean ematen dute, nabarmen, beste senideek baino denbora gehiago, eta beste senideek baino astialdirako tarte urriagoa izaten dute. Ebidentziak nabarmentzen dira zaintzaren denbora eta ordaindutako lanaren arteko lotura negatiboa aztertzean, eta, horretaz gain, euren denboraren erabileraz galdetzean, balorazio ezkorragoa egiten dute zaintzaile-rola betetzen dutenek.
\end{abstract}

\section{HITZ-GAKOAK:}

zaintza, menpekotasuna, ezgaitasunak, adinduak, denboraren erabilera.
Este artículo aborda el tiempo de cuidado y sus efectos en la vida cotidiana de quienes conviven con personas mayores en su hogar. A través de información procedente de la Encuesta de Empleo del Tiempo 2002-2003, se intenta responder a las siguientes preguntas: ¿cómo se reparte el cuidado en los hogares con personas mayores dependientes?, ¿se elige cuidar de manera libre?, ¿cuánto tiempo se dedica al cuidado? y ¿qué efectos tiene el cuidado en el uso y la vivencia del tiempo de los cuidadores? Este trabajo revela intensas desigualdades en el reparto de las responsabilidades de cuidado. Los resultados demuestran que los cuidadores dedican significativamente más tiempo a tareas domésticas y tienen menos tiempo libre que el resto de personas de su hogar. Se ofrecen evidencias de que existe una relación negativa entre tiempo de cuidado y tiempo de trabajo remunerado, y de que los cuidadores experimentan su tiempo cotidiano de manera más negativa que quienes no cuidan.

\section{PALABRAS Clave:}

cuidado, dependencia, discapacidad, personas mayores, uso del tiempo.

${ }^{1}$ Una gran parte de este artículo está basada en el libro Los tiempos del cuidado. El impacto de la dependencia de los mayores en la vida cotidiana de sus cuidadores (Jesús Rogero García, Imserso, 2010). Los datos empíricos aquí mostrados proceden de este trabajo, salvo que se especifique lo contrario. 


\section{La necesidad de medir el cuidado}

El cuidado no remunerado a personas dependientes se ha reconocido en las últimas décadas como una actividad fundamental desde el punto de vista social y económico (Durán, 2002), y ha generado muchas y variadas investigaciones en el ámbito español e internacional. Desde ese redescubrimiento del cuidado (Bazo, 1998; Hunter y McPerson, 1993), producido a finales de los años setenta, en esta área de investigación han confluido múltiples disciplinas y perspectivas, desde la ética a la economía, desde la salud a la sociología, y se ha producido un indudable avance en el conocimiento de su realidad. En el caso de España y de acuerdo con la Encuesta de Discapacidad, Autonomía Personal y Situaciones de Dependencia (INE, 2008), el cuidado familiar cubre al $93 \%$ de las personas dependientes por discapacidad mayores de 4 años que viven en hogares, mientras que la atención de profesionales privados alcanza al $14 \%$, y los servicios públicos, sólo al $7 \%$ (Rogero-García, 2011). Dada la gran intensidad que suele requerir el cuidado, la mayor parte de los estudios en este campo han abordado sus efectos negativos sobre los propios cuidadores familiares. No obstante, recientemente ha emergido con fuerza una corriente que pone en valor el proceso de cuidado y reflexiona sobre sus aspectos positivos (Fast, 1999; Cohen, Colantonio y Vernich, 2002), enfatizando su carácter intrínseco a la naturaleza humana (Martín Palomo, 2010) y cuestionando la idea de dependencia y normalidad (Rodríguez y Ferreira, 2010; Toboso y Guzmán, 2010).

Las situaciones de dependencia constituyen un área de investigación en expansión que requiere de anclajes empíricos para conocer mejor su realidad. A pesar de los avances en este campo, aún queda mucho por conocer sobre la dimensión cuantitativa del cuidado y, especialmente, sobre sus efectos en la vida cotidiana de los cuidadores (Bittman et al., 2004) ${ }^{2}$. Generalmente, el cuidado se ha medido a través del tiempo dedicado (por los cuidadores), y del tiempo requerido o recibido (por las personas dependientes). Ambos indicadores son fundamentales para el conocimiento de esta realidad. En el uso del tiempo, se reflejan los lazos sociales, las desigualdades socioeconómicas, las interdependencias personales y los recursos con los que se hace frente a las necesidades y preferencias. El tiempo es tan variado como las actividades que realizan los individuos. Puede ser un deleite o una tortura, libremente elegido o impuesto, podemos regalarlo o pedirlo

${ }^{2}$ La investigación internacional sobre el tiempo de cuidado (y sobre uso del tiempo, en general) se caracteriza por una gran variedad metodológica y por la dificultad de comparar diferentes países y regiones. Captar el uso del tiempo es una empresa difícil y, si se pretende hacer de manera precisa, requiere de una gran cantidad de recursos (Durán y Rogero, 2009). prestado. El estudio del uso del tiempo de individuos y grupos constituye un acercamiento preciso a sus limitaciones y opciones vitales. En este sentido, el uso del tiempo no ha sido suficientemente estudiado en relación con la calidad de vida y el bienestar, cuestiones que hoy constituyen una interesante y prolífica área de estudio (a este respecto, y en relación con la salud de las personas mayores, vid. Prieto-Flores et al., 2008), y que se vinculan directamente con el cuidado.

El objetivo de este artículo es responder, a través de información empírica sencilla, a las siguientes preguntas: ¿cómo se reparte el cuidado en los hogares con personas dependientes?, ¿por qué se produce ese reparto?, ¿cuánto se cuida? y ¿qué efectos tiene la atención en el uso y vivencia del tiempo? Para ello, se exponen parte de los resultados de una investigación más amplia (Rogero-García, 2010) de manera sintética. La mayoría de la información procede de la Encuesta de Empleo del Tiempo 20022003 (en adelante, EET 2002-2003) del Instituto Nacional de Estadística. La reciente Encuesta de Empleo del Tiempo realizada por el INE (2009-2010) no permite realizar un análisis de estas características (INE, 2009), puesto que ha eliminado la pregunta relativa a dificultades en la realización de actividades de la vida diaria y, por tanto, también la posibilidad de identificar la relación de cuidado en el hogar (quién cuida a quién). La EET 2002-2003 utiliza el diario de actividades, considerado como la metodología con más validez y fiabilidad para el estudio del uso del tiempo (Eurostat, 2008).

\section{Factores y motivos para cuidar}

¿Quién dona su tiempo para dedicarlo a cuidar y por qué lo hace? La distribución del cuidado en los hogares no es, ni mucho menos, equitativa: como es sabido, cuidan más las mujeres que no tienen empleo (o lo tienen reducido) y a partir, aproximadamente, de 55 años (Imserso, 2005). No está tan claro, sin embargo, en qué medida cuida más este grupo y cuáles son los factores que refuerzan la distribución desigual de las responsabilidades de atención.

A este respecto, es especialmente relevante el modo en que se produce la decisión de cuidar. De acuerdo con datos de la Encuesta de Apoyo Informal a las Personas Mayores en España 2004 (Imserso), el $36 \%$ de los cuidadores no eligen cuidar de manera directa, sino que lo hacen a raíz de condicionantes externos, y sólo en el $5 \%$ de los casos la persona dependiente escoge al cuidador. Las mujeres tienen más probabilidades de cuidar como consecuencia de una decisión familiar, y los hombres suelen hacerlo más por iniciativa propia y cuando no hay otros familiares en su entorno que puedan ocuparse de ello. A mayor cercanía en la relación de parentesco y menor severidad de la discapacidad, más pro- 
babilidad de asumir el cuidado por iniciativa propia. En este aspecto, el cuidado no se asume en un contexto de libertad, porque, frecuentemente, existe algún tipo de coerción. Como es obvio, en los casos en los que el cuidado no es libremente elegido, la frustración y el estrés de los cuidadores son mayores, y la satisfacción con la actividad es sensiblemente menor.

Estos procesos de toma de decisiones desembocan en la asignación de responsabilidades de cuidado, es decir, en quién asume el cuidado en la familia. Buena parte de las investigaciones sobre cuidado familiar han consistido en describir las características de los cuidadores, generalmente a través del contraste entre un grupo de cuidadores y el resto de la población. Este procedimiento, sin embargo, no tiene en cuenta si el resto de la población está o no expuesta a demandas de cuidado, con lo que la comparación entre ambos grupos está distorsionada de partida. Si se quiere identificar los factores que intervienen en que el cuidado sea asumido por una persona concreta, es crucial analizar sólo aquellos contextos en los que varias personas podrían asumirlo, es decir, aquellas personas expuestas a la misma demanda de cuidado.

Con ese fin, en este estudio se seleccionaron sólo aquellos hogares en los que había varias personas que podrían cuidar33. Asimismo, se controló el número de personas del hogar, variable que establece probabilidades diferentes a priori (en los hogares en los que conviven tres personas - una de ellas dependiente- la probabilidad de cuidar será, a priori, del $50 \%$ para cada uno de los miembros no dependientes, y así sucesivamente), a través de un modelo de regresión logística multinivel.

En este análisis, las mujeres tenían casi seis veces más probabilidades de cuidar que los hombres (tabla 1). Incluso en hogares con seis miembros, las mujeres tenían más del $50 \%$ de probabilidades de cuidar, en contraste con un $15 \%$ de probabilidad para los varones. Los miembros del hogar sin empleo tuvieron 4,5 veces más probabilidades de cuidar que los miembros con empleo, un resultado que indica tres posibilidades: a) que las personas cuidaban porque estaban sin empleo (en el momento de la aparición de la dependencia del familiar estaban 'disponibles' para cuidar), b) que no tenían

${ }^{3}$ La identificación de las personas con discapacidad se realizó a partir de la pregunta "¿Le impide alguna enfermedad crónica física o mental, incapacidad o deficiencia desarrollar su actividad diaria?", con tres posibles respuestas: "Sí, intensamente", "Sí, hasta cierto punto" y "No". La elección de cualquiera de las dos primeras categorías indicaba discapacidad, cuya intensidad se dedujo de las categorías "intensamente" y "hasta cierto punto" (INE, 2003). Se consideraron cuidadores aquellas personas del mismo hogar que dedicaron al menos 10 minutos al día a "ayudas a adultos miembros del hogar" o "trayectos debidos al cuidado de adultos del hogar". empleo porque cuidaban (decidieron no buscar empleo, o dejar el que tenían, para cuidar), o c) ambas cosas. Que el modelo seleccione la relación con el empleo y el sexo confirma que ambos factores tienen efectos por separado, es decir, que las mujeres no cuidan más sólo por su situación respecto al empleo, sino también por el hecho de ser mujeres.

Tabla 1. Análisis multinivel de la probabilidad de cuidar en el hogar. España 2002-2003

\begin{tabular}{l|c|c|c}
\hline & Coeficiente & $\begin{array}{c}\text { Desviación } \\
\text { estándar }\end{array}$ & T-ratio \\
\hline Constante & 0,626 & 0,470 & 1,334 \\
\hline Tamaño del hogar & $-0,550$ & 0,093 & $-5,925$ \\
\hline Varones & - & - & - \\
\hline Mujeres & 2,010 & 0,345 & 5,819 \\
\hline No ocupado & - & - & - \\
\hline Ocupado & $-1,318$ & 0,292 & $-4,519$ \\
\hline Otra situación & - & - & - \\
\hline Entre 18 y 39 años, & 1,154 & 0,309 & 3,730 \\
\hline o más de 64 & - & - & - \\
\hline Entre 40 y 64 años & 1,319 & 0,324 & 4,066 \\
\hline
\end{tabular}

N: 409 personas (211 cuidadores; 198 no cuidadores); 186 hogares. Porcentaje clasificado correctamente: $80 \%$. Especificidad: $77,8 \%$. Sensibilidad: $82,1 \%$.

Fuente: Rogero-García (2010), a partir de microdatos de la Encuesta de Empleo del Tiempo 2002-2003 (INE).

El análisis específico de los cónyuges reveló que el $18 \%$ de los cónyuges varones no cuidaban a sus mujeres dependientes, porcentaje que se reducía al $2 \%$ entre las mujeres cónyuges ${ }^{4}$. Estos resultados confirman que los varones desempeñan, en muchos casos, papeles secundarios en el cuidado de sus esposas (Jiménez-Martín y Vilaplana, 2008), debido a su más avanzada edad, a su menor preparación para afrontar el rol de cuidador (por atribuciones de género durante su experiencia vital) y a que reciben más apoyo de otros familiares y de cuidadoras pagadas que las mujeres en la misma situación. Asimismo, también pueden existir reticencias por parte de las mujeres dependientes a ser ayudadas por sus maridos (Roberto, 1999), especialmente si hay otras alternativas. Entre los descendientes de las personas dependientes, las hijas tenían casi cuatro veces más probabilidades de cuidar que sus hermanos, y 13 veces más cuando quien requería los cuidados era la madre.

\section{Cuánto y cuándo se cuida}

Una vez analizado quién cuida en los hogares, es conveniente estimar la cantidad de atención que se provee. El cuidado familiar se desarrolla generalmente a diario (Imserso, 2005a). De acuerdo con la

4 Estos resultados corresponden a modelos que, por cuestiones de espacio, no reproducimos en este artículo. Pueden consultarse en detalle en Rogero-García (2010). 
EET 2002-2003, los cuidadores que conviven en el hogar de la persona dependiente dedican un promedio de 1,59 horas diarias a ayudas físicas directas (comer, levantarse de la cama, asearse) 5 . Estos cuidadores invierten algo menos tiempo los fines de semana que los días laborables ( 1,40 y 1,75 horas, respectivamente), probablemente porque es el momento en que reciben más ayudas de familiares de fuera del hogar. No obstante, el cuidado no se limita únicamente al apoyo en actividades básicas de la vida diaria, sino que también consiste en actividades instrumentales, tales como la limpieza del hogar y la preparación de alimentos, y en actividades avanzadas de la vida diaria, como el apoyo para actividades de ocio o las relaciones familiares (Fillembaum, 1995). Ocuparse de una persona dependiente implica, más allá de los cuidados físicos directos, un considerable aumento del trabajo no remunerado en el hogar, cuyo reparto es fundamental en la distribución de las responsabilidades reales de cuidado.

Los miembros de los hogares en los que convive al menos una persona dependiente dedican, en conjunto, un promedio de 9 horas al día a trabajo no remunerado (tareas domésticas y cuidado). Los cuidadores realizan, como promedio, el $71,2 \%$ de esas tareas, y quienes no cuidan realizan el $13,7 \%$. Esta diferencia revela la gran desigualdad en la distribución del trabajo doméstico en el hogar, incluyendo la atención a personas dependientes. El número de miembros del hogar es, obviamente, clave en ese reparto de estas tareas: cuantas más personas, más posibilidades de repartirlo, pero también más trabajo que realizar. A pesar de que la proporción del trabajo no remunerado realizado por el cuidador se reduce en hogares con más miembros, su tiempo de dedicación es mayor con cada miembro añadido (gráfico 1).

Es llamativo que, en los hogares en los que conviven únicamente la persona dependiente y su cuidador, las cuidadoras acumulen más del $90 \%$ del trabajo no remunerado, mientras que los hombres cuidadores en la misma situación realicen el $67 \%$. Cuando los cuidadores asumen un porcentaje alto del trabajo no remunerado del hogar, tienden a buscar ayudas externas, ya sea de otros familiares o de profesionales remunerados. La probabilidad de contar con estas ayudas es mayor entre los cuidadores varones y entre los descendientes de la persona dependiente.

5 Esta actividad fue definida en el diario de actividades de la EET 2002-2003 como "ayudas a adultos miembros del hogar", que equivale a aquella "asistencia a adultos y cuidado de los mismos (excepto trabajos domésticos)"; y más concretamente, a los "cuidados físicos administrados a un adulto enfermo o a una persona de edad avanzada: aseo, corte de pelo, masajes; ayuda psíquica, información y asesoramiento; acompañar a un adulto al médico, visitarlo en un hospital” (INE, 2003: 94).

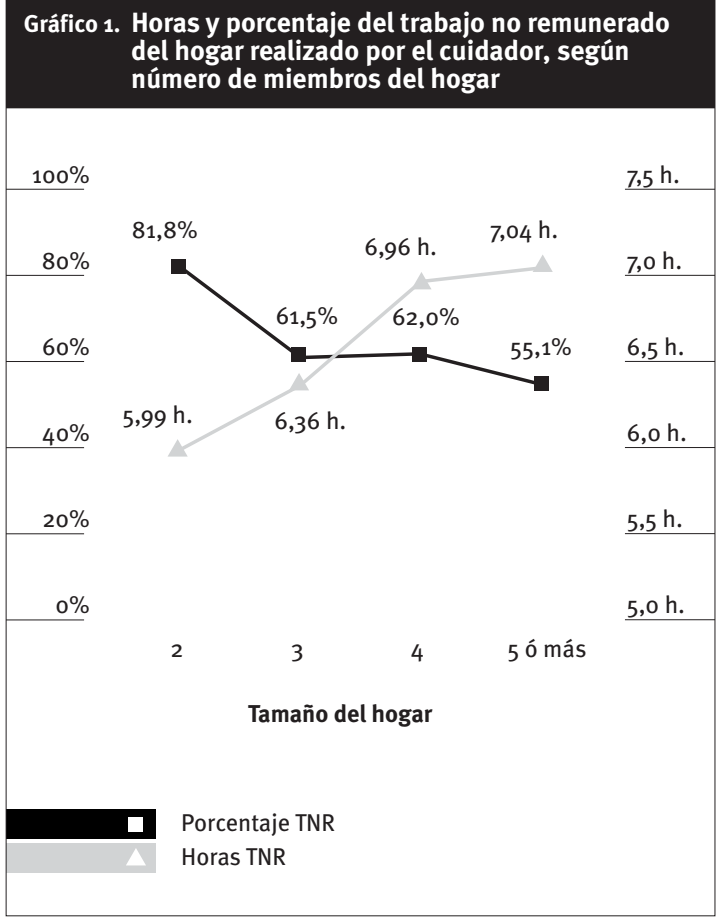

Fuente: Rogero-García (2010), a partir de microdatos de la Encuesta de Empleo del Tiempo 2002-2003 (INE).

La división de responsabilidades en el interior de los hogares parece clara: quien asume el cuidado también se ocupa del resto de las actividades domésticas. Así, en muchos hogares se produce una especialización en actividades de mercado o del hogar, que presumiblemente persigue la eficiencia en la ejecución de las tareas (Becker, 1981). Sin embargo, la teoría de la especialización productiva no alcanza a explicar por qué, en los hogares en los que hombres y mujeres no están vinculados al mercado de trabajo, los primeros dedican mucho menos tiempo a actividades domésticas que las mujeres. Tras estas diferencias parecen esconderse cuestiones sociales y culturales que poco o nada tienen que ver con la eficiencia económica.

La cantidad de tiempo dedicado a cuidado y actividades complementarias ofrece una visión parcial de esta actividad, puesto que no revela cuándo se desarrolla ni si la prestación es continuada o se da en diferentes momentos del día. La realidad del cuidado exige a los cuidadores sincronizar su propio tiempo con las necesidades de la persona dependiente, a través de, en ocasiones, una compleja planificación temporal que armonice los tiempos familiares y los del empleo. Durante el día, es más frecuente proveer cuidado entre las $8: 30 \mathrm{~h}$ y las 11:00 h (algo más tarde los sábados y domingos) y en las horas centrales de la tarde (gráfico 2). No resulta sencillo saber si el momento en que se cuida responde a las necesidades de las personas dependientes 0 a las de sus cuidadores. Las primeras 


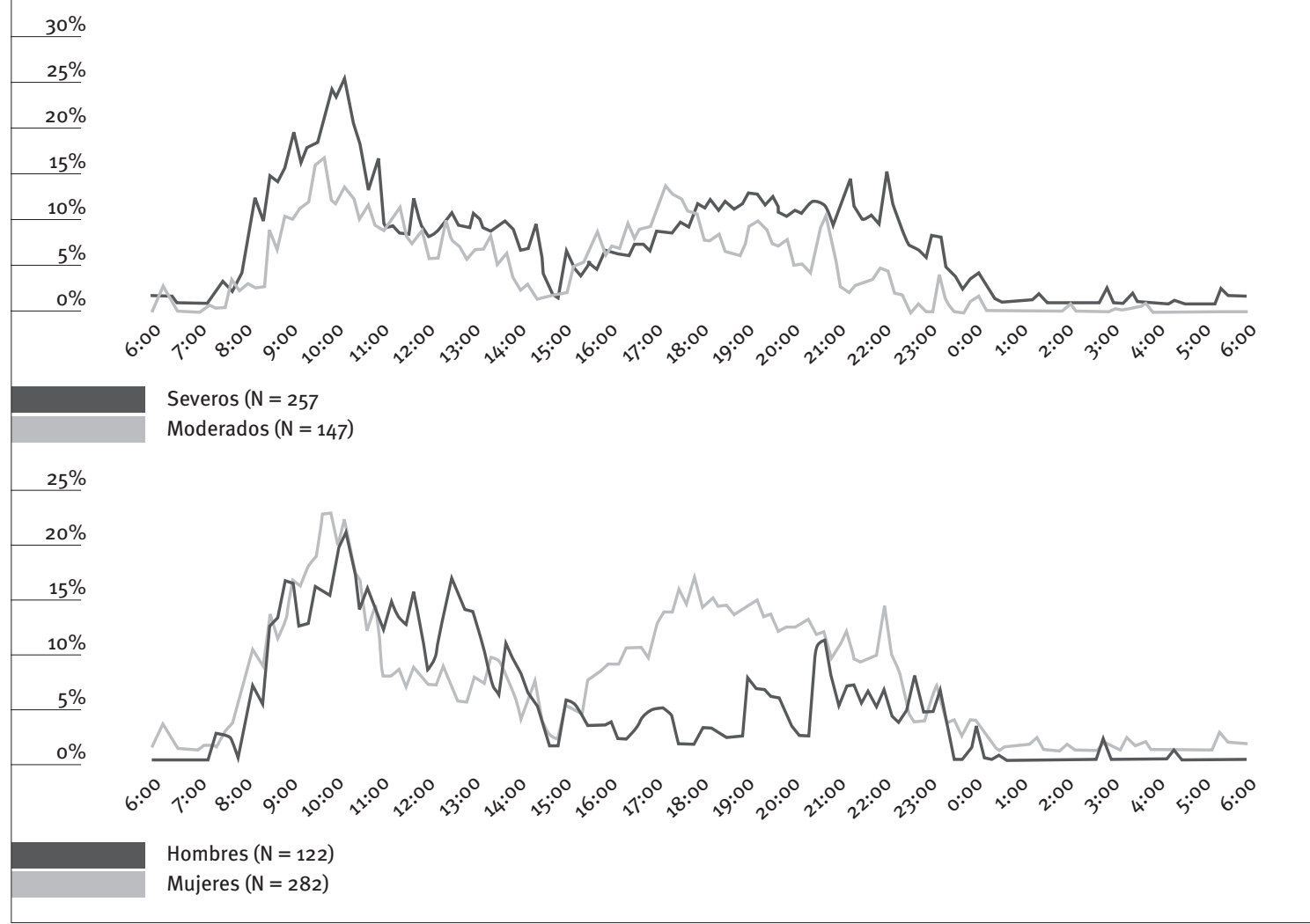

Fuente Rogero-García (2010), a partir de microdatos de la Encuesta de Empleo del Tiempo 2002-2003 (INE).

horas de la mañana constituyen el momento en que los mayores pueden requerir apoyo para levantarse, vestirse 0 asearse, $y$, probablemente, las horas de la tarde están más disponibles para los cuidadores, al verse libres de sus obligaciones laborales.

Quienes cuidan de personas con mayor grado de dependencia dedican más tiempo y lo hacen de manera más continuada durante el día. Asimismo, los hombres cuidan en mayor proporción por las mañanas, mientras que el cuidado de las mujeres está presente a lo largo de todo el día. Probablemente, esta pauta de cuidado se relaciona con el vínculo cuidador-receptor: los hombres cuidan de sus esposas sobre todo cuando están jubilados, de manera que su atención se concentra en las mañanas, mientras que por la tarde es más probable que reciban apoyo de otros familiares (hijas, fundamentalmente). Por otro lado, algunas discapacidades concretas requieren dedicación durante la noche: el $8,7 \%$ de los cuidadores provee atención entre las 00:00 h y las 07:00 h, lo cual repercute de manera importante en el tiempo de descanso de los cuidadores (Casey, 2004). Se ha estimado que, para el caso de patologías concretas, como la demencia, este porcentaje puede aumentar hasta el $40 \%$ (Rivera Navarro, 1999).

\section{Qué implica cuidar: los efectos sobre la vida cotidiana}

Las encuestas sobre uso del tiempo permiten ampliar la visión del análisis de una actividad concreta, como es el cuidado, al estudio de la distribución del tiempo de los cuidadores en las diferentes actividades (Bittman et al., 2004). De este modo, es posible obtener una visión global de la vida cotidiana (lo que ha sido denominado 'perfil temporal') de quienes conviven con personas dependientes y, ulteriormente, estimar los efectos del cuidado sobre los cuidadores.

Un modo de hacerlo es comparando la distribución del tiempo diario en actividades de tres grupos: $a$ ) los cuidadores, b) quienes no cuidan pero conviven con personas dependientes, y c) quienes no conviven con personas dependientes. Estos tres grupos dedican cantidades de tiempo muy diferentes a trabajo remunerado y no remunerado: los cuidadores dedican menos de una hora diaria al empleo e invierten más de seis horas en trabajo no remunerado (gráfico 3). El cuidado constituye el $27 \%$ del trabajo no remunerado que realizan diariamente. Quienes conviven con personas dependientes y no cuidan invierten más de cuatro horas al día en traba- 
jo remunerado, y su dedicación a actividades domésticas es de menos de 2 horas, muy por debajo no sólo de los cuidadores, sino también de la población general que no convive con personas dependientes. El perfil temporal del cuidador es, por tanto, muy diferente al del resto de la población.

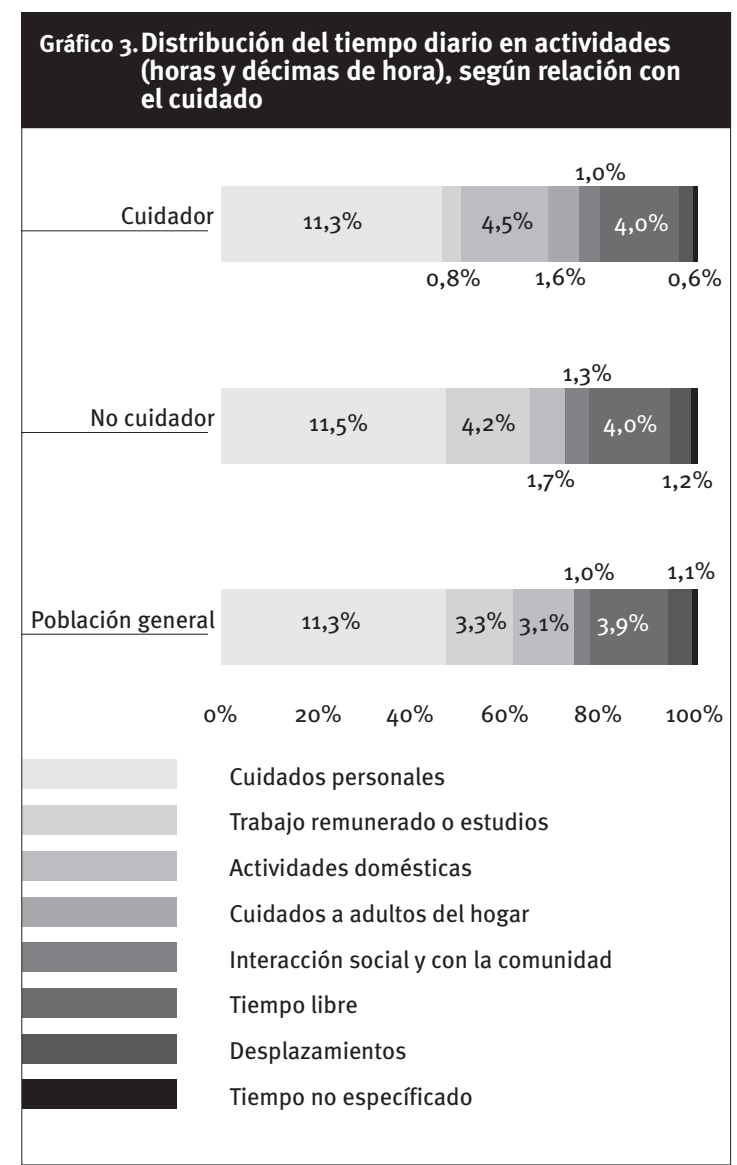

N: 404 cuidadores, 198 no cuidadores, 40.129 población general. Fuente: Rogero-García (2010), a partir de microdatos de la Encuesta de Empleo del Tiempo 2002-2003 (INE).

A pesar de que hombres y mujeres dedican un tiempo muy similar a cuidar $(1,5$ y 1,6 horas, respectivamente), el resto de actividades refleja un uso del tiempo muy diferente. Los cuidadores varones invierten en actividades domésticas menos de la mitad de tiempo que las cuidadoras $-2,5$ y 5,4 horas, respectivamente- (gráfico 4 ). Los hombres cuidadores responden a las demandas de atención fundamentalmente a través de cuidados físicos directos, y no tanto con ayuda en actividades domésticas, mientras que las mujeres atienden ambos tipos de necesidades. El tiempo libre revela la experiencia del cuidado para ambos sexos: los cuidadores varones disponen diariamente de casi cinco horas y media, dos horas más que las cuidadoras.

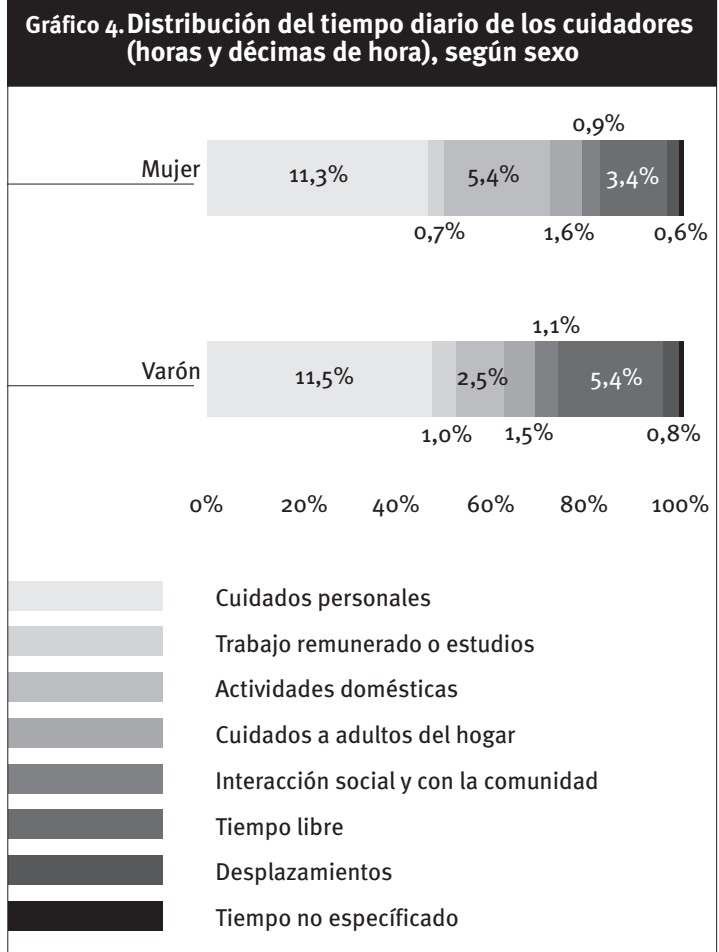

N: 122 varones y 282 mujeres.

Fuente: Rogero-García (2010), a partir de microdatos de la Encuesta de Empleo del Tiempo 2002-2003 (INE).

Las diferencias según sexo son incluso más abultadas entre los cuidadores con empleo. Las cuidadoras ocupadas dedican al cuidado prácticamente lo mismo que el resto de cuidadoras ( 1,5 horas), mientras que los cuidadores varones ocupados reducen sensiblemente su dedicación (0,9 horas, un $40 \%$ menos que quienes no tienen empleo). Las cuidadoras ocupadas dedican al resto de actividades domésticas 3,9 horas diarias, dos horas y media más que los cuidadores con empleo. Por su parte, éstos invierten casi una hora más en trabajo pagado (4 y 4,9 horas, respectivamente). La carga global de trabajo -suma de trabajo remunerado y no remunerado- (García Sainz, 1999) de las cuidadoras ocupadas es de 9,4 horas diarias, mientras que la de los cuidadores ocupados es de 7,3 horas. Estos últimos disponen de 1,3 horas diarias más de tiempo libre. Los datos dejan patente que las mujeres experimentan una mayor repercusión temporal a causa del cuidado, independientemente de su relación con el mercado de trabajo.

Un modo de calcular el efecto del cuidado en el uso del tiempo es comparar las actividades de los cuidadores ocupados (ambos sexos) con las del resto de ocupados (población general). Es razonable suponer que estas diferencias revelarán el tiempo añadido/sustraído atribuible a las demandas de atención. De acuerdo con estos supuestos, los cuidadores ocupados dedican un $27 \%$ menos de tiem- 
Tabla 2. Diferencias de tiempo dedicado a actividades por los ocupados que cuidan y por los ocupados que no cuidan (horas y décimas de hora)

\begin{tabular}{|c|c|c|c|c|}
\hline & A & B & C & D \\
\hline & $\begin{array}{c}\text { Ocupados población } \\
\text { general }\end{array}$ & $\begin{array}{l}\text { Ocupados } \\
\text { cuidadores }\end{array}$ & $\begin{array}{l}\text { Diferencia } \\
\text { (A-B) }\end{array}$ & $\begin{array}{l}\text { Porcentaje } \\
(C * 100 / 2)\end{array}$ \\
\hline Cuidados personales & 10,7 & 10,7 & 0,0 & 0,0 \\
\hline Trabajo remunerado o estudios & 5,8 & 4,3 & $1,5^{\star \star}$ & 27,1 \\
\hline Actividades domésticas & 2,1 & 3,1 & $-1,0^{\star \star}$ & $-49,9$ \\
\hline Cuidado a adultos del hogar & 0,0 & 1,3 & $-1,3^{\star \star}$ & - \\
\hline Interacción social y con la comunidad & 0,9 & 1,0 & $-0,1$ & $-10,7$ \\
\hline Tiempo libre & 2,9 & 2,5 & 0,4 & 14,7 \\
\hline Desplazamientos & 1,4 & 0,9 & $0,5^{\star \star}$ & 32,9 \\
\hline Tiempo no especificado & 0,1 & 0,2 & $-0,1$ & $-59,5$ \\
\hline
\end{tabular}

* Significativo al 0,05. ** Significativo al 0,01.

Fuente: Rogero-García (2010), a partir de microdatos de la Encuesta de Empleo del Tiempo 2002-2003 (INE).

po a trabajo pagado como consecuencia del cuidado, un $50 \%$ más a actividades domésticas (sin incluir el cuidado), un $15 \%$ menos a tiempo libre y un $33 \%$ menos a desplazamientos (tabla 2).

El hecho de estar a cargo de una persona en situación de dependencia no incide únicamente en el tiempo de los cuidadores, sino también en la configuración de su espacio cotidiano. Los estudios en este campo han revelado que, frecuentemente, la dependencia obliga a transformar el espacio físico y los modos de convivencia en el hogar, con el fin de prepararlos para el uso intensivo que implica el cuidado (Yantzi, 2005; Durán, 2005). Asimismo, es habitual que los cuidadores permanezcan en el hogar no sólo para proporcionar cuidados físicos, sino también para supervisar a la persona dependiente y actuar en caso necesario. A pesar de los avances en monitorización a distancia a través de las nuevas tecnologías (telecuidado y telemedicina), muchas veces las necesidades de las personas dependientes son urgentes y requieren respuestas inmediatas, algo que difícilmente se puede abordar sin una presencia constante en el hogar.

Los cuidadores pasan un promedio de 19,6 horas diarias en sus hogares, de modo que están 4,4 horas fuera del hogar, más de tres horas menos que el resto de la población adulta (7,5 horas) [EET 20022003]. El tiempo en el hogar de quienes cuidan a personas con discapacidad severa es superior al de quienes cuidan de personas con discapacidad moderada (20,2 horas y 18,7 horas, respectivamente). Este aspecto también muestra la diferencia en las condiciones de cuidado de mujeres y hombres: las primeras pasan 2,3 horas diarias más en el hogar que los varones cuidadores. La alta permanencia en el hogar es consecuencia de la realización de una serie de actividades que fueron definidas por Montgomery et al. (1985) como "tareas que confinan" (confining tasks). En muchas ocasiones, cuando los cuidadores salen del hogar, lo hacen para realizar actividades asociadas con la atención (gestiones, compra de alimentos) y deben buscar a alguien que supervise a la persona dependiente.
Los cuidadores experimentan, de este modo, restricciones en su libertad tanto en lo relativo al uso del tiempo como en el espacio cotidiano que habitan. Esta situación tiene consecuencias psicológicas y sociales: el $20 \%$ de los cuidadores se sienten agobiados muy frecuentemente, frente al $11 \%$ de la población general y al 7,8\% de las personas que viven con personas dependientes pero no cuidan (EET 2002-2003). Los cuidadores de personas con discapacidades más severas se sienten agobiados con mayor frecuencia que quienes cuidan personas con discapacidad moderada. La percepción de agobio y de escasez de tiempo también es más intensa entre los cuidadores con empleo que entre los cuidadores sin empleo y que entre quienes tienen empleo y no cuidan.

\section{Del tiempo expropiado al tiempo donado}

Este artículo proporciona una visión panorámica de la situación de quienes prestan cuidados a personas mayores de su hogar, desde el punto de vista de sus actividades y vivencia cotidiana, con el objetivo último de mostrar algunas de las consecuencias no visibles del cuidado. A través de información de diferentes encuestas, especialmente de la EET 2002-2003, se han identificado los factores asociados con la asignación de responsabilidades de cuidado, los efectos del cuidado en el uso del tiempo y la vivencia subjetiva de esos efectos.

En los últimos años, amplios sectores académicos y sociales han demandado a las instituciones públicas una mayor dotación y articulación de servicios para apoyar a las familias en sus responsabilidades de cuidado. La llegada de la crisis económica en 2008 ha traído consigo una potente corriente ideológica que ha planteado la necesidad ineludible de recortar derechos sociales, incluidos los previstos en la Ley de Dependencia (39/2006), debido a su elevado coste económico. Sin embargo, y como se ha puesto de manifiesto de manera repetida, la mirada exclusivamente monetarista sobre el papel de los servicios 
públicos carece de la amplitud necesaria para servir de referencia a la toma de decisiones políticas. Por ello, se requieren análisis que muestren los costes, en términos de bienestar social y de calidad de vida, de la falta de servicios de apoyo a las personas dependientes y sus familias. Desde nuestro punto de vista, esas consecuencias, muchas de ellas ocultas, no han tenido la atención que merecen y, consecuentemente, el debate sobre la atención a personas dependientes no se ha fundamentado en la realidad del fenómeno de manera suficiente.

En la experiencia de cuidar a un ser querido, quienes cuidan pueden experimentar su propio tiempo como un tiempo robado -expropiado por las circunstancias o por los demás-, o como un tiempo ofrecido desinteresada y afectuosamente. El cuidado contiene así dos vertientes que pueden anularse en función de la situación. Los tiempos de atención elevados, los altos grados de dependencia, la escasez de apoyos formales e informales, y las situaciones económicas precarias suelen transformar lo que podría ser una experiencia positiva y enriquecedora en una vivencia angustiosa y frustrante del cuidado.

Los resultados de este trabajo reflejan una distribución muy desigual de las responsabilidades de atención y de sus efectos negativos, que es consecuencia de una toma de decisiones familiar en ocasiones conflictiva e injusta, y cuyo origen está en la desigualdad de poder entre los miembros de la familia. Muy probablemente, el cuidado a personas dependientes no constituiría un problema social si se repartiera equitativamente y en un contexto de libertad de elección. Debemos promover que la atención a los seres queridos no se convierta en un tiempo expropiado, sino en un tiempo libremente donado. Se trata de conservar el tiempo de cuidado como un bien precioso, porque preservar el cuidado es preservar lo más humano (Boff, 2002). Un tiempo que debe ser distribuido en términos de justicia, atendiendo a criterios de bienestar, y gestionado de manera eficiente y eficaz, sin prisas innecesarias, $\sin$ penurias y $\sin$ angustias. 


\section{Bibliografía}

BAZO, M. T. (1998): “Vejez dependiente, políticas y calidad de vida”, Papers, № 56, págs. 143-161.

BECKER, G. S. (1981): A Treatise on the Family, Cambridge; Massachusetts; Harvard, University Press.

BITTMAN, M. et al. (2004): "Making the invisible visible: The life and time(s) of informal caregivers", en BITTMAN, M. y FOLBRE, N. (eds.): Family Time: The Social Organisation of Care, Londres; Nueva York, Routledge, págs. 69-89.

BOFF, L. (2002): El cuidado esencial: ética de lo humano, compasión por la tierra, Madrid, Trotta.

COHEN, C. A.; COLANTONIO, A.; y VERNICH, L. (2002): "Positive aspects of caregiving: Rounding out the caregiver experience", International Journal of Geriatric Psychiatry, vol. 17, $\mathrm{n}^{0} \mathbf{2}$, págs. 184-188.

DURÁN, M. A. (dir.) [2005]: Informe sobre el impacto social de los enfermos dependientes por ictus. Informe ISEDIC, 2004, Madrid, Merck Sharp; Dohme España.

- (2002): Los costes invisibles de la enfermedad, 2aㅡ edición, Bilbao, Fundación BBVA.

DURÁN, M. A.; y ROGERO GARCÍA, J. (2009): La investigación sobre el uso del tiempo, Madrid, Centro de Investigaciones Sociológicas.

EUROSTAT (2008): Harmonised European Time Use Surveys, Eurostat, Luxemburgo.

FAST, J. (1999): “Informal Caregiving: Is it Really Cheaper?”, en International Association of Time Use Researchers Conference, Colchester.

FILLENBAUM, G. G. (1995): “Activities of daily living”, en MADDOX, G. (ed.): The Encyclopedia of Aging, $2^{\text {a }}$ edición, Nueva York, Springer, págs. 7-9.
GARCÍA SÁINZ, C. (1998): “La carga global de trabajo. Un análisis sociológico". Tesis doctoral. Departamento de Cambio Social (Sociología I), Universidad Complutense de Madrid.

HUNTER, D.; y MACPHERSON, I. (1993): “Influencia de los cuidadores informales sobre la provisión de servicios y las decisiones de asignación", en JAMIESON, A.; e ILLSLEY, R. (eds.): Comparación de políticas europeas de atención a las personas ancianas, Barcelona, SG Editores; Fundación de Caja de Madrid.

IMSERSO (2005): Cuidados a las personas mayores en los hogares españoles. El entorno familiar, Madrid, Instituto de Mayores y Servicios Sociales.

INE (2009): Encuesta de Empleo del Tiempo 2009-2010. Proyecto, Madrid, Instituto Nacional de Estadística.

- (2003): Proyecto metodológico de la Encuesta de Empleo del Tiempo 2002-2003, Madrid, Instituto Nacional de Estadística.

JIMÉNEZ-MARTÍN, S.; y VILAPLANA, C. (2008): “Trade-off between formal and informal care in Spain", Documentos de Trabajo FEDEA, 끙. 22.

MARTÍN-PALOMO, M. (2010): “Autonomía, dependencia y vulnerabilidad en la construcción de la ciudadanía”, Zerbitzuan, vol. 48, págs. 57-69.

MONTGOMERY, R. J. V.; GONYEA, J. G.; y HOOYMAN, N. R. (1985): "Caregiving and the experience of subjective and objective burden", Family Relations, vol. 34, págs. 19-26.

PRIETO-FLORES, M. E. et al. (2008): “Factores sociodemográficos y de salud en el bienestar emocional como dominio de calidad de vida de las perso- 
nas mayores en la Comunidad de Madrid 2005", Revista Española de Salud Pública, vol. 82, no 3 , págs. 301-313.

RIVERA, J. (1999): “Redes familiares en el cuidado del anciano con demencia. Análisis evolutivo de un estudio poblacional”. Tesis Doctoral. Departamento de Sociología III, Universidad Complutense de Madrid.

ROBERTO, K. A. (1999): "Making critical health care decisions for older adults: Consensus among family members", Family Relations, vol. 48, no 2, pags. 167-175.

RODRÍGUEZ, S.; y FERREIRA, M. (2010): “Diversidad funcional: sobre lo normal y lo patológico en torno a la condición social de la dis-capacidad", Cuadernos de Relaciones Laborales, vol. 28, $\mathrm{n}^{0} \mathbf{1}$, págs. 151-172.

ROGERO-GARCÍA, J. (2011): “El cuidado a las personas dependientes por discapacidad en España y el papel de las familias, a examen", en CASADO, D. (coord.): Acción y políticas de apoyo a las familias. Crianza, atención a la dependencia y renovación demográfica, Hacer Editorial (en prensa).

- (2010): Los tiempos del cuidado. El impacto de la dependencia de los mayores en la vida cotidiana de sus cuidadores, Madrid, Instituto de Mayores y Servicios Sociales.

TOBOSO, M.; y GUZMÁN, F. (2010): “Cuerpos, capacidades, exigencias funcionales... y otros lechos de Procusto", Política y Sociedad, vol. 47, nํㅜ 1, págs. 67-83.

YANTZI, N. M. (2005): "Balancing and negociating the home as a place of caring: The experiences of families caring for children with long-term care needs". Tesis Doctoral. Department of Geography, Queen's University (Ontario, Canadá). 